\title{
Human spermatozoa anti-proprotein convertase subtilisin/kexin type 4 synthesis using New Zealand rabbit for novel immunocontraception in males
}

\author{
Dahril ${ }^{1}$, Aulanni'am ${ }^{2}$, Vivi Keumala ${ }^{3}$, Akhmad Mustafa $^{4}$ \\ Departments of ${ }^{1}$ Urology and ${ }^{3}$ Clinical Pathology, Faculty of Medicine, Universitas Syiah Kuala, Dr. Zainoel Abidin General Hospital, Banda Aceh, ${ }^{2}$ Department of Science, \\ Universitas Brawijaya, Malang, ${ }^{4}$ Department of Urology, Faculty of Medicine, Universitas Padjadjaran, Hasan Sadikin Hospital, Bandung, Indonesia
}

Purpose: Proprotein convertase subtilisin/kexin type 4 (PCSK4, a 54-kDa protease) is expressed in the plasma membrane of the acrosome in human spermatozoa. It plays a critical role in penetrating the zona pellucida. Synthesis of human anti-PCSK4 might be important for novel male immunocontraception.

Materials and Methods: We used semen from adult males as the source of antigen (acrosomal PCSK4). Isolation and antigen characterization were done by immunohistochemistry followed by electrophoresis. Purification of PCSK4 was done by the electroelution method, followed by immunization with an animal model (Oryctolagus cuniculus). Antibody was collected, purified, and tested by using Western blot and dot blot. Antibody in vitro potential testing was performed on human spermatozoa by laser scanning microscopy with rhodamine stain under a light microscope and on rat spermatozoa.

Results: Human anti-PCSK4 bound with PCSK4 on the head of human spermatozoa and could interfere with rat spermatozoa activity to penetrate the oocyte.

Conclusions: The result of this study can be used as a basis to develop new immunocontraceptives targeted towards males. This study shows that antibodies from induction of PCSK4 from spermatozoa may hinder fertilization.

Keywords: Antibodies; Contraception, immunologic; Proprotein convertases

This is an Open Access article distributed under the terms of the Creative Commons Attribution Non-Commercial License (http://creativecommons.org/licenses/by-nc/4.0) which permits unrestricted non-commercial use, distribution, and reproduction in any medium, provided the original work is properly cited.

\section{INTRODUCTION}

Overpopulation is a global issue that leads to limited resources. Nowadays more than $75 \%$ of all couples worldwide use various methods of contraception, with most contraception targeted at females [1]. Various methods of male contraception are well known for their inconvenience, irreversibility, and failure [1].

Immunocontraception is a safe, effective, and most importantly, reversible new method of contraception for males. This contraceptive method leads to the generation of a humoral and/or cell-mediated immune response against

Received: 7 January, 2019 - Accepted: 4 April, 2019

Corresponding Author: Akhmad Mustafa

Department of Urology, Faculty of Medicine, Universitas Padjadjaran, Hasan Sadikin Hospital, Jl. Pasteur No. 38, Jawa Barat, Bandung 40161, Indonesia TEL: +62-811232827, FAX: +62-651-52053, E-mail: mustafa.urologi@gmail.com

ORCID: https://orcid.org/0000-0001-5631-9174 
antigens that play a critical role in the reproductive process. These vaccines can be designed to inhibit the (1) production of gametes (spermatozoa and oocyte), (2) functions of gametes (obstructing fertilization), and (3) gamete outcome (pregnancy) [2].

Human spermatozoa has become a focus of study for immunocontraceptive development since the discovery of antisperm antibody (ASA) in 2\% to $30 \%$ of infertile couples and in $70 \%$ of post-vasectomy males [3]. Various forms of spermatozoa-based immunocontraception have been studied before, such as fertilin $\alpha$ (ADAM 1), fertilin $\beta$ (ADAM 2 ), cyritestin (ADAM 3), hyaluronidase (PH-10), lactate dehydrogenase-C4, sperm antigen-10, fertilization antigen-1, and epididymal protease inhibitor, but none of these can produce total fertility inhibition in animal models [4-6].

Proprotein convertase subtilisin/kexin type 4 (PCSK4) is also known as proprotein convertase type 4 and is one nine family members of the calcium-dependent serine endoproteinases, which play a role in proteolytic activation of secretory precursor proteins into bioactive protein forms. PCSK4 molecules are found in testicular germ cells and spermatozoa. PCSK4 $54 \mathrm{kDa}$ is one of the specific molecules in males found only along the plasma membrane of the spermatozoa acrosome. Thus, if its activity is inhibited, it will not interfere with the function of other cells or organs [7].

In this study we aimed to describe the steps of producing anti-PCSK4 from human spermatozoa as antigen by using New Zealand rabbit (Oryctolagus cuniculus) to produce antibodies as well as in vitro antibody potential testing.

\section{MATERIALS AND METHODS}

In this study we used sperm from an adult, healthy, fertile human male. The sperm sample was obtained via self-ejaculation. After collection of the sperm, we began processing the sperm to isolate PCSK4. Laboratory processing to isolate the PCSK4 protein was carried out in several steps:

1. Confirmation of PCSK4 protein in the acrosomal plasma membrane by immunohistochemistry.

2. Isolation of human PCSK4 protein by electrophoresis and specificity evaluation by Western blot (Figs. 1, 2) [8-12].

3. Purification of PCSK4 protein by the electroelution method (Fig. 3) [8].

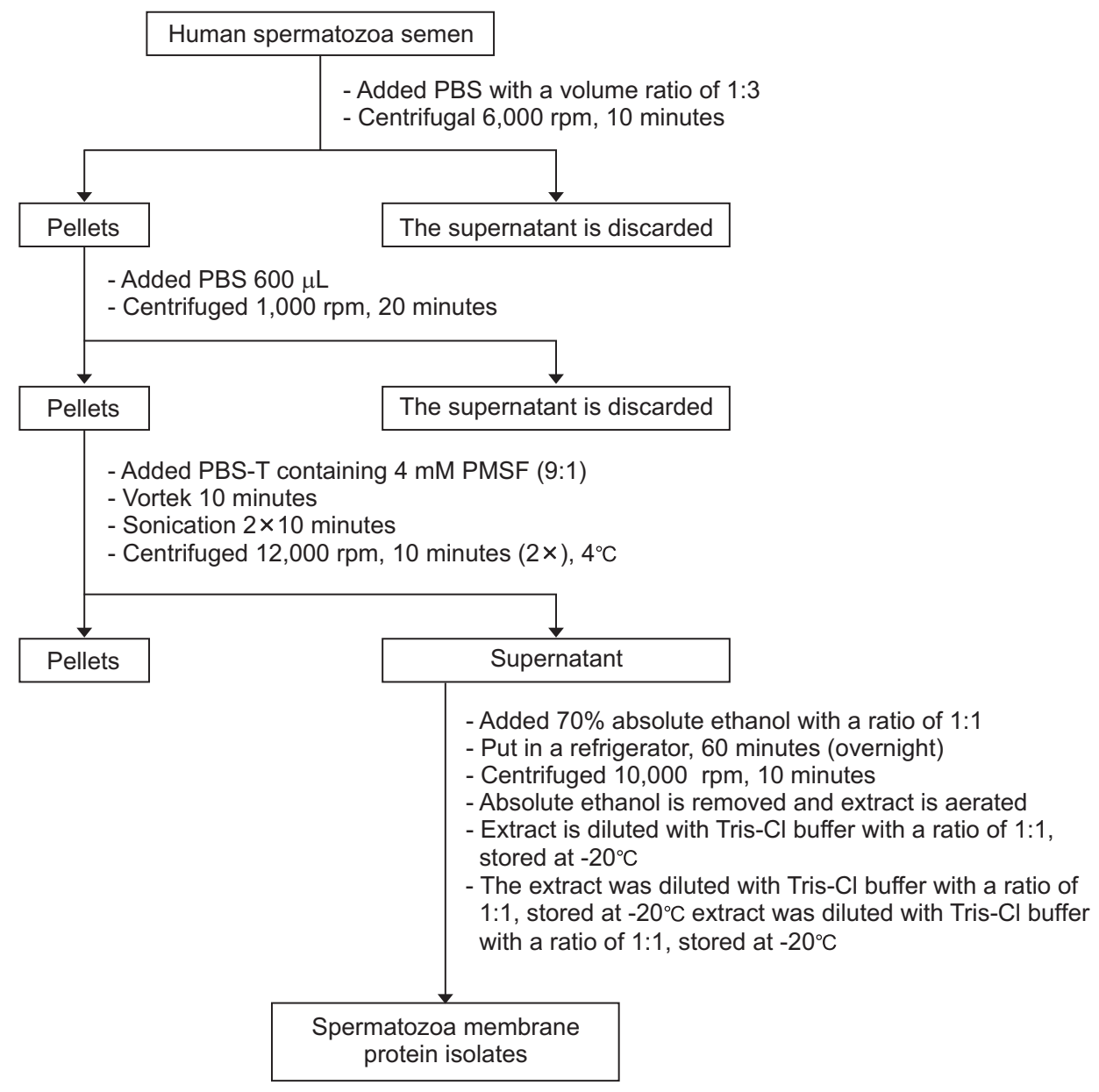

Fig. 1. Isolation of 54-kDa protein in human spermatozoa membrane. PBS, phosphate-buffered saline; PBS-T, PBSTween 20; PMSF, phenylmethylsulfonyl fluoride. 
Antibody production was done by using adult New Zealand rabbits (O.cuniculus) aged 9 to 12 months. We used three adult male rabbits. Because rabbits are larger than mice, more antibody is produced than if we used mice as the model. The PCSK4 enzyme molecule was added with complete Freund's Adjuvant (CFA) at a ratio of 1:1 and a volume of $150 \mu \mathrm{L}$. The enzyme levels of PCSK4 used were 20

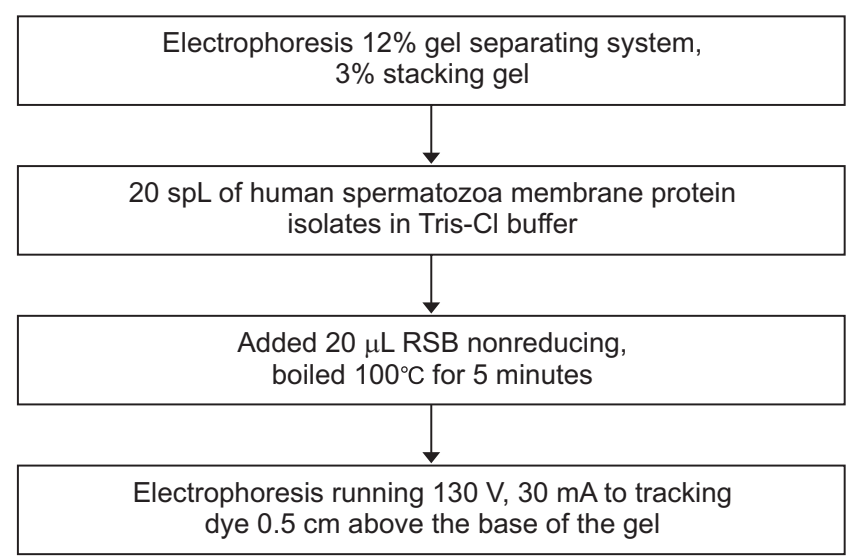

Fig. 2. Sodium Dodecyl Sulphate-Polyacrylamide Gel Electrophoresis (SDS-PAGE) electrophoresis of human spermatozoa protein membrane. RSB, reducing sample buffer. to $50 \mathrm{ng}$. The mixture was homogenized by using a Guo Hua Vortex to form a stable emulsion $[8,10]$.

The rabbits were injected with human spermatozoal PCSK4 in an intraperitoneal adjuvant (CFA) for the first immunization. The second (day 21) and third (day 57) immunizations were performed by using the $54-\mathrm{kDa}$ protein in incomplete Freund's Adjuvant in the same way. Control rabbits were immunized with phosphate-buffered saline for the first, second, and third immunization.

Rabbit blood was taken from the auricular vein in the ear by using a disposable 3-mL syringe. Blood collection was carried out on days 29, 36, 43, 50, 57, 64, 71, 78, 85, and 92 after the first immunization. The blood samples then underwent several processes to isolate and purify the antibody:

1. Serum anti-PCSK4 purification (Fig. 4).

2. Human spermatozoa PCSK4 polyclonal antibody characterization in the animal model using an indirect enzyme-linked immunosorbent assay (ELISA) method.

3. Specificity testing of anti-PCSK4 $54 \mathrm{kDa}$ against PCSK4 $54 \mathrm{kDa}$ antigen by dot blot and Western blot (Figs. 5, $6)$.

After specificity testing, we performed human spermatozoa anti-PCSK4 in vitro potential testing for its receptor

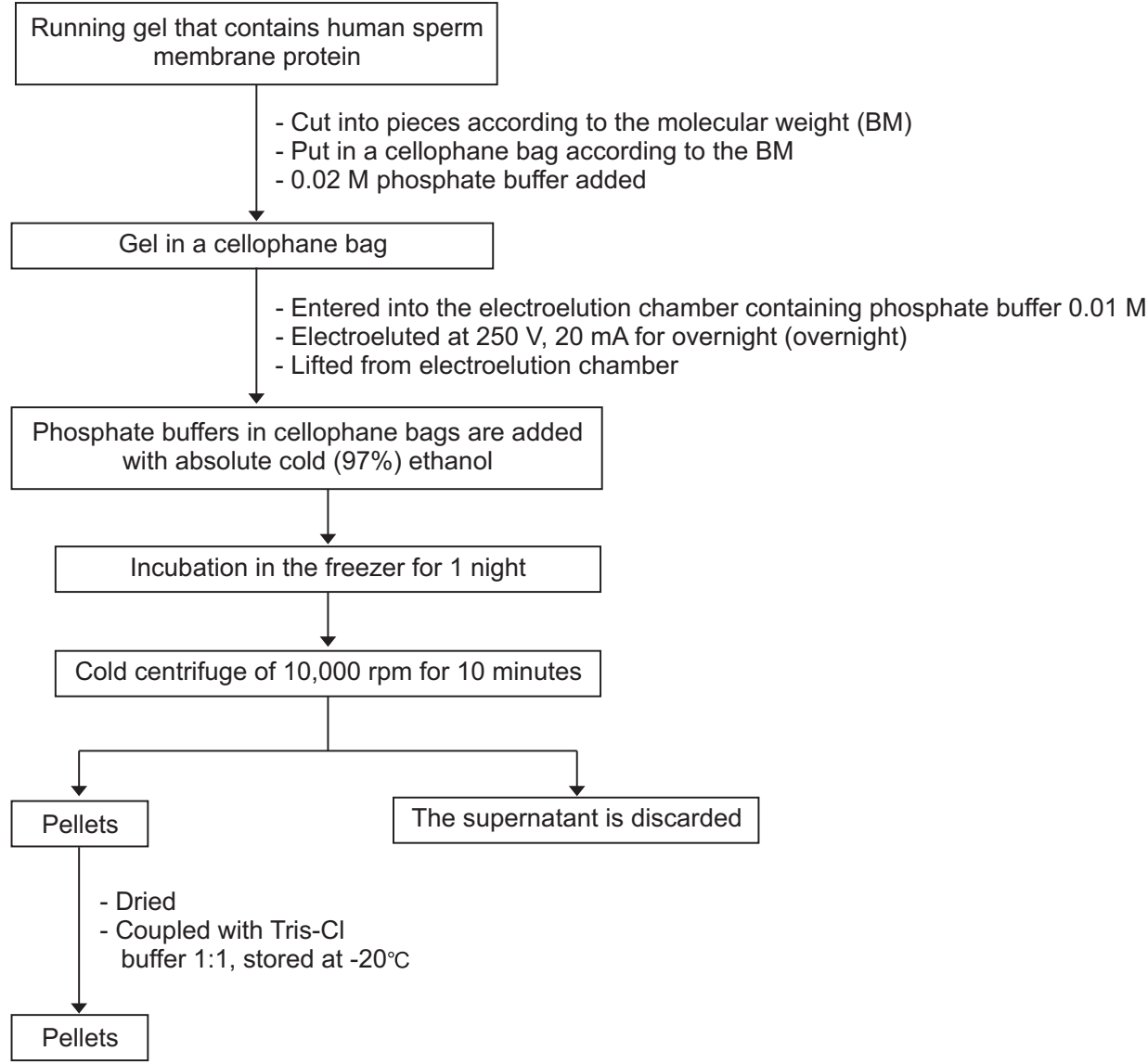

Fig. 3. Purification of proteins by electroelution method. 


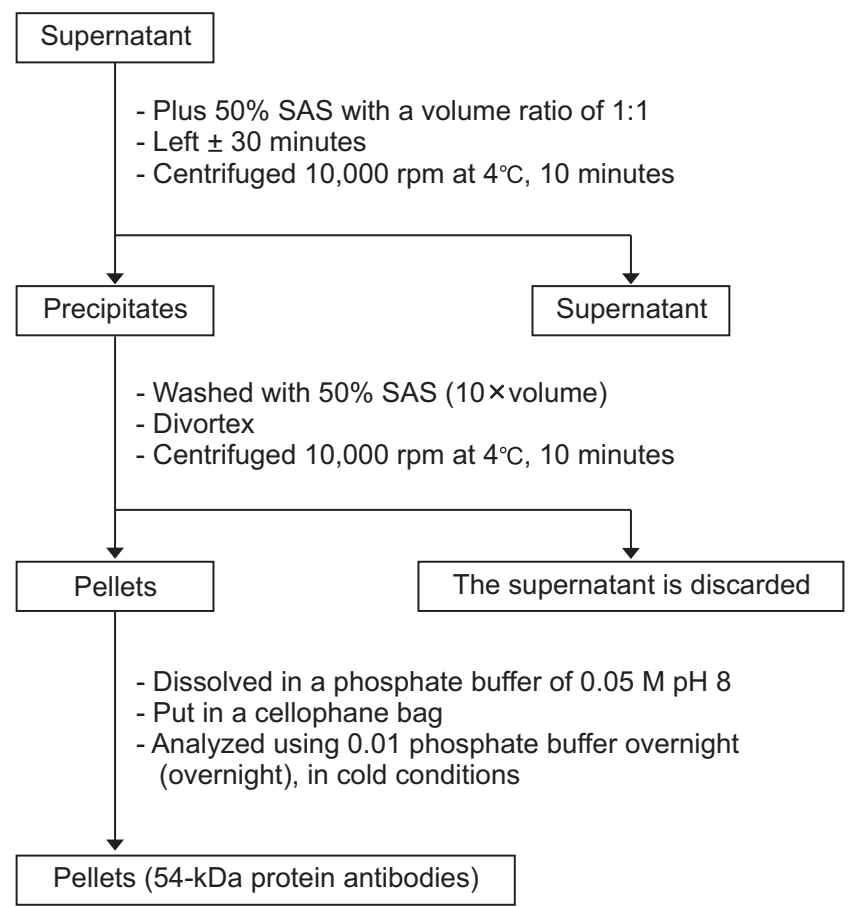

Fig. 4. Purification of serum antibody. SAS, supercritical antisolvent.

in the acrosomal plasma membrane of human spermatozoa. Observation using laser scanning microscopy (LSM) with rhodamine stain revealed PCSK4 $54 \mathrm{kDa}$ in human spermatozoa. To determine the potency of the antibody for inhibiting penetration of the oocyte, we used oocyte and spermatozoa from rats (Rattus norvegicus) and observed them under a microscope. The rat spermatozoa used in this study were within the normal ranges of morphology, sperm count, and motility.

The sperm donors for this study had been informed and agreed to donor their sperm for this research and signed the informed consent form. This study was conducted in the Biochemical and Bioscience Laboratory, Universitas Brawijaya, Malang, and received ethical clearance from the ethical committee of Universitas Brawijaya (approval number: 458/UN6.C21.2/KEPK/PN/2013; Institutional Animal Care: Institut Biosains Universitas Brawijaya Malang). All study subjects were treated according to animal welfare guidelines according to William Russel and Rex Burch [13,14]

\section{RESULTS}

\section{PCSK4 antigen isolation}

Immunohistochemistry methods were used to confirm PCSK4 protein in the acrosomal plasma membrane to ensure that the source of the protein was from human spermatozoa (Fig. 7). Characterization of human spermatozoa PCSK4

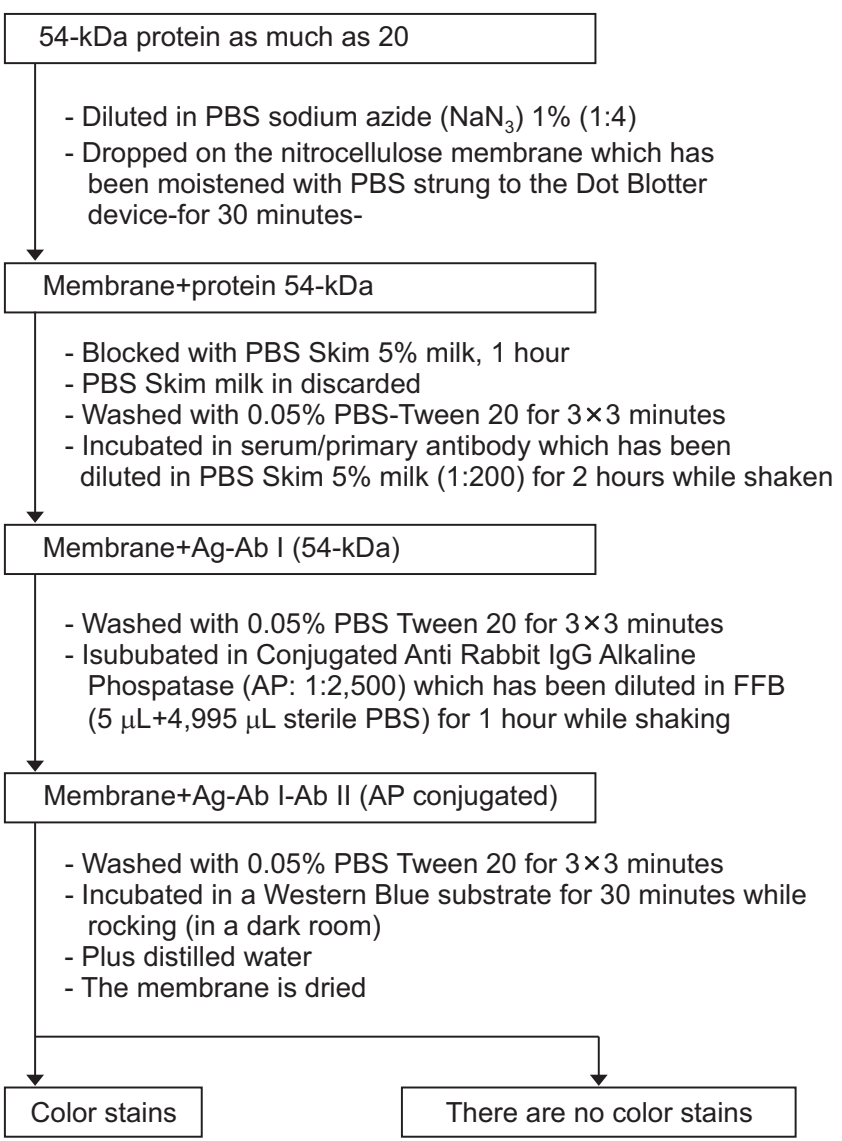

Fig. 5 . Step by step sequence for performing specificity test using dot blotting. PBS, phosphate-buffered saline; IgG, immunoglobulin G; FFB, final formulation buffer; AP, alkaline phosphatase.

polyclonal antibody in the animal model was done by use of the indirect ELISA method. As shown by ELISA, the mean concentration of PCSK4 in human spermatozoa was 701,838 $\mathrm{ng} / \mathrm{m}$.

\section{Anti-PCSK4 characterization}

Rabbit blood was collected and processed according to the study methods mentioned earlier. Using indirect ELISA, we found the highest titer of anti-PCSK4 at an optical density of 0.312 and 0.335 (405 nm wavelength). Specificity testing of anti-PCSK4 $54 \mathrm{kDa}$ against PCSK4 $54 \mathrm{kDa}$ antigen was performed by Western blot and dot blot. The results obtained were compared on the basis of membrane color (Fig. 8). Western blot and dot blot proved that anti-PCSK4 from human spermatozoa could specifically bind with PCSK4 protein.

\section{Anti-PCSK4 in vitro potency test}

We performed antibody potency testing using rhodamine staining to prove that the anti-PCSK4 from human spermatozoa could bind with PCSK4 protein on human sper- 


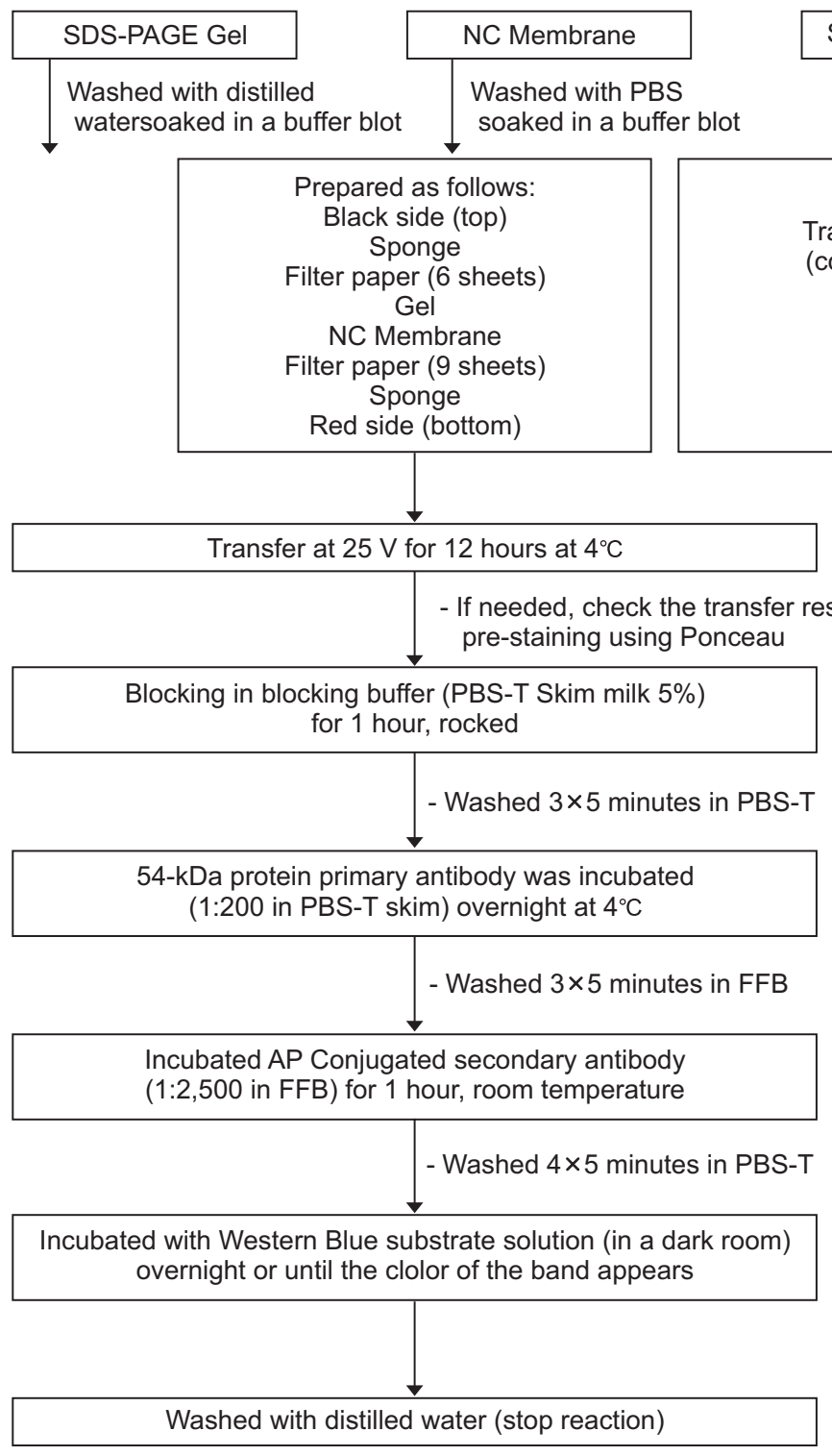

matozoa. The results shown by LSM indicated that antiPCSK4 could bind on the head of human spermatozoa (Fig. 9). To determine the antibody potency in inhibiting penetration of the oocyte, we used oocyte and spermatozoa from rats ( $R$. norvegicus). As observed under a microscope, rat spermatozoa that had been added with human antiPCSK4 could not recognize its receptor in the rat oocyte. The rat spermatozoa moved away from the oocyte and could not penetrate the oocyte (Fig. 10).

\section{DISCUSSION}

This research was conducted in four stages, most of which were explorative. As such, we conducted no statistical analysis except for the in vivo test, and the data were presented descriptively in pictures and graphs.
Sponge, filter paper Soaked in a blot buffer

Transfer conditions (constant voltage):

$\mathrm{V} \times$ hour $=300$

$25 \times 12=300$

$20 \times 15=300$

$50 \times 6=300$
Fig. 6 . Step by step sequence for specificity test using Western blot method. SDS-PAGE, Sodium Dodecyl SulphatePolyacrylamide Gel Electrophoresis; NC, nitrocellulose; $\mathrm{PBS}$, phosphate-buffered saline; PBS-T, PBS-Tween 20; AP, alkaline phosphatase; $F F B$, final formulation buffer.
PCSK4 protein was confirmed in the plasma membrane of human spermatozoa by using PCSK4 antibodies and immunocytochemical methods. PCSK4 protein was immunocytochemically shown to be expressed on the plasma membrane of human spermatozoa. The study published by Gyamera-Acheampong [5] and Gyamera-Acheampong and Mbikay [15] reported similar findings: the PCSK4 molecule is expressed in germ cells and is located along the acrosomal plasma membrane of mammalian spermatozoa. Other researchers such as Bergeron et al. [16] and Torii et al. [17] found the presence of PCSK4 molecules in reproductive organs, especially the testes, and in germ cells in the form of spermatocytes and round spermatids. Other studies also mention that PCSK4 can be detected in ejaculate of human sperm and that this profile can be used as a marker of sperm quality. 
The PCSK4 molecule is a proteolytic enzyme with an important role in reproductive physiology. Disruption of the proteolytic activity of this enzyme can cause fertility disorders [15]. This study proves that the protein produced by spermatozoa ejaculate contains PCSK4 enzyme as measured by the sandwich ELISA method using the Human PCSK4 ELISA kit (cat. no. CSB-EL017643HU). This result is evidence that PCSK4 found in spermatozoa ejaculate can be selected as a ligand binding receptor marker for fertilization and that anti-PCSK4 can be developed as an immunocontraceptive candidate in males capable of binding to PCSK4 on the plasma membrane of the spermatozoa acrosome so as to inhibit fertilization in the ovum cell.

Characteristics of PCSK4 54-kDa protein isolated from human spermatozoa include a molecular weight of 54

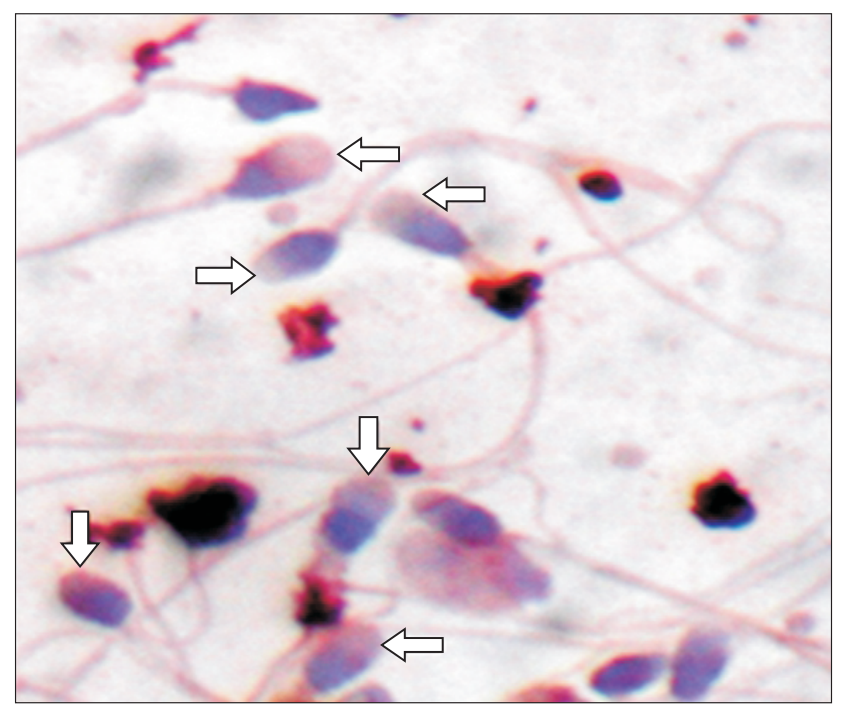

Fig. 7. Proprotein convertase subtilisin/kexin type 4 (PCSK4) expression on the surface of spermatozoa plasma membrane. Arrows: PCSK4 expression on the acrosome (immunohistochemistry method using $D A B$ chromogen, counterstain using methyl green, $\times 100$ ).
$\mathrm{kDa}$, which was assessed by Sodium Dodecyl SulphatePolyacrylamide Gel Electrophoresis and confirmed by Western blotting. This is consistent with the results of several studies in mammals showing that PCSK4 molecules are found in acrosomal granules, acrosomal ridges, and spermatozoa acrosomal plasma membrane surfaces $[15,18]$. Research by Gyamera-Acheampong [5] and GyameraAcheampong and Mbikay [15] found the PCSK4 molecule in the spermatozoa acrosomal plasma membrane in cauda epididymis of the rat.

The results in the present study using Western blotting showing PCSK4 in ejaculate of human spermatozoa can be developed into immunocontraception in males through the production of PCSK4 human antibodies. This concept was tested in vitro and in vivo. The bond between PCSK4 and its antibodies causes a conformational change in the spermatozoa cell membrane protein that is ejaculated, causing an acrosome reaction disturbance, and spermatozoa will not recognize their receptors in ovum cells [15].

The 54-kDa PCSK4 protein band in this study was collected by the electroelution method and used as an antigen to produce polyclonal antibodies in a rabbit model $(O$. cuniculus), which will be used for the development of male immunocontraception. The protein level of PCSK4 $54 \mathrm{kDa}$ used as an immunogen to produce PCSK4 antibody $54 \mathrm{kDa}$ in rabbits was $701,838 \mathrm{ng} / \mathrm{mL}$. The rabbits were then used as male contraceptive candidates in rat models ( $R$. norvegicus). The results of the protein content of PCSK4 isolated from human spermatozoa have the potential to test the inhibition of fermentation in vitro and in vivo according to studies carried out by Aulani [10] and Kurniawan [8]

The results from indirect ELISA in the present study showed that PCSK4 $54 \mathrm{kDa}$ from spermatozoa could cause an immune response in an animal model. This immune response resulted in the production of PCSK4 $54 \mathrm{kDa}$
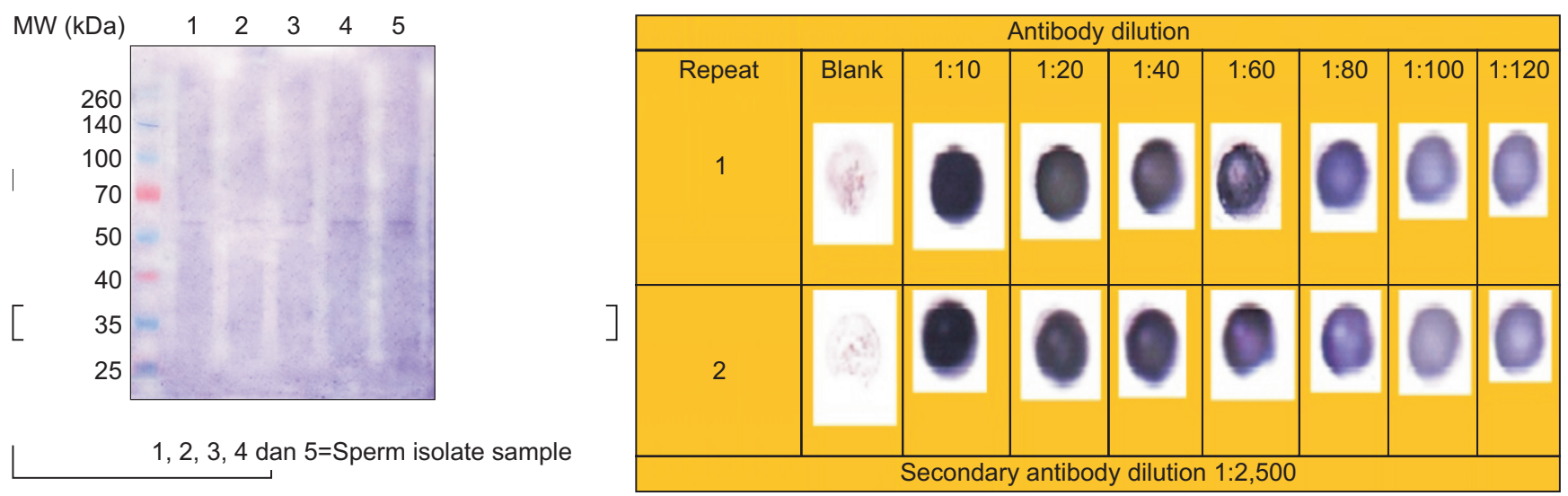

Fig. 8. Specificity test of anti-proprotein convertase subtilisin/kexin type 4 (PCSK4) to PCSK4 using Western blot and dot blot technique. 

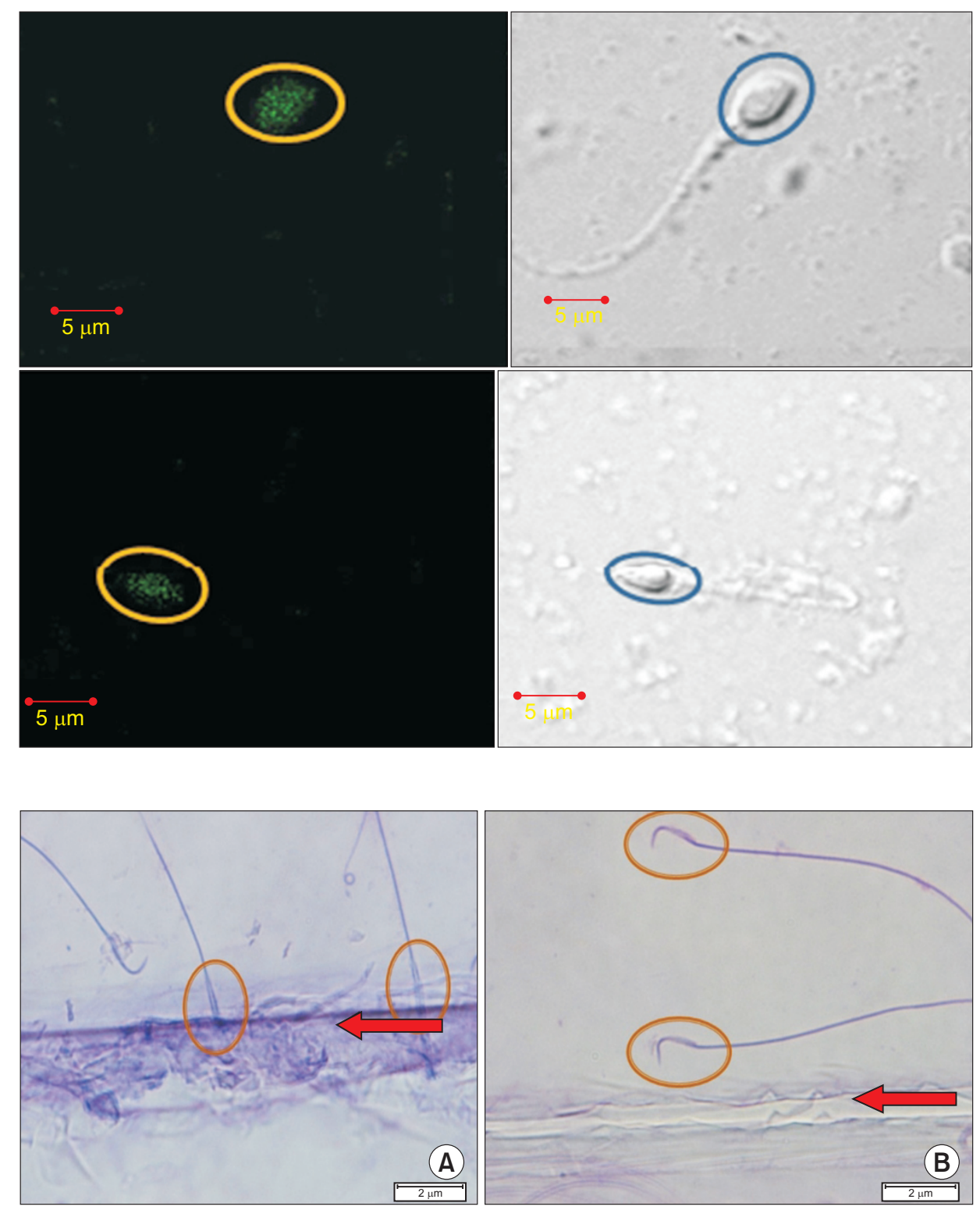

Fig. 9. Ligand binding receptor (proprotein convertase subtilisin/kexin type 4 [PCSK4] with human anti-PCSK4) with confocal laser scanning microscope (CLSM) examination. Green color (yellow circles) is PCSK4 bound with anti-PCSK4 on the head of human spermatozoa. Blue circles highlight the normal appearance of the spermatozoa head.

Fig. 10. Spermatozoa receptor and oocyte binding inhibition. (A) Rat spermatozoa without anti-proprotein convertase subtilisin/kexin type 4 (PCSK4) immunization can move and bind to oocyte extract on the object glass. (B) Rat spermatozoa with anti-PCSK4 immunization move away from the oocyte extract. Arrows: rat oocyte extract on the object glass. Circles explain that the spermatozoa move away from the oocyte. antibodies. An increase in titers in the third post-booster blood collection reflects an increase in the number of antibodies as the primary immune response to fight against the first antigen exposure. The number of antibodies will decrease and increase again after the booster. Post-booster titers have a higher value than before (primary immune response). The titer profile after immunization has the same tendency to reach a peak after the first and second booster. This increase is a result of a secondary immune response involving memory B cells. The primary immune response activates B cells natively, whereas B cells natively produce antibodies to respond to antigen entry for the first time.

The primary immune response will produce the highest antibody titers around the 14th day and then will experience a decrease in antibody titers. The secondary immune response involves memory B cells. Memory B cells proliferate faster than native B cells. The secondary immune response takes place faster and produces more antibodies than the primary immune response. In the primary immune response, $B$ cells differentiate into plasma cells and produce more immunoglobulin (Ig) M than IgG. The secondary immune response produces more IgG, IgA, and IgE [18]. The measured IgG titer in this examination is believed to be due to the response of PCSK4, which is an immunogenic molecule with a molecular weight of $54 \mathrm{kDa}$.

The test of PCSK4 antibody specificity can be seen as a purplish blue stain in the dot blot (Fig. 8). The purplish blue color gradation indicates variations in concentration in the PCSK4 antigen reaction with PCSK4 antibodies. The anti-PCSK4 molecule, which is diluted to 1:120, can still be recognized by PCSK4 antigen. This positive reaction shows that PCSK4 isolated from spermatozoa can induce anti- 
PCSK4. The results of this study showed that PCSK4 is an immunogenic molecule capable of inducing a humoral immune response, namely IgG (the antibody produced by its induction) identified by its immunogen, PCSK4 [10]. As stated by previous researchers, spermatozoa have antigenic properties [19].

According to Protocol Online 109, the dot blot test provides information about the number of bonds between antigens and antibodies based on color intensity. The higher the intensity of the color, the greater the concentration of the reaction between antigens and antibodies. Stains with varying color intensities show that there is a variation in the strength of the reaction between the antigen (PCSK4) and its antibody, anti-PCSK4.

The results of the Western blot also showed the specificity of PCSK4 $54 \mathrm{kDa}$ antibody to PCSK4 $54 \mathrm{kDa}$. The Western blot results indicated that the PCSK4 $54 \mathrm{kDa}$ molecule bound specifically to the PCSK4 $54 \mathrm{kDa}$ antibodies produced in rabbits (O. cuniculus).

Based on the test results of the specificity of the reaction in the Western blot, it can be believed that the IgG titer measured in the indirect ELISA method, and also the dot blot, is the response of the PCSK4 molecule with a molecular weight of $54 \mathrm{kDa}$, which is immunogenic. This is consistent with the theory that proteins composed of amino acids having a molecular weight of more than $10 \mathrm{kDa}$ can induce immune reactions (i.e., are immunogenic) [20,21].

The spermatozoa receptor binding test in this study is an in vitro test using human spermatozoa, where PCSK4 $54 \mathrm{kDa}$ as the antigen receptor is attached to the acrosomal plasma membrane. This study proved the existence of a ligand binding receptor between PCSK4 antigen $54 \mathrm{kDa}$ in spermatozoa and PCSK4 antibody $54 \mathrm{kDa}$ as the result of induction in the rabbit model (O. cuniculus). Fig. 9 shows the binding on the membrane area of the spermatozoa head. This is consistent with the study of Burkin and Miller [22], which showed that the zone of protein binding occurs in the spermatozoa acrosome. This also shows that the PCSK4 54 $\mathrm{kDa}$ molecule is attached to the spermatozoa head plasma membrane [15]. Other studies also suggest that the acrosome is located in the anterior part of the spermatozoa head, and in humans makes up $40 \%$ to $70 \%$ of the spermatozoa head [23].

We found evidence of a ligand bond between PCSK4 antibody $54 \mathrm{kDa}$ and PCSK4 $54 \mathrm{kDa}$ on the plasma membrane of the spermatozoa acrosome. If this enzyme cannot function in proteolytic processes, it can cause disturbances in acrosome reactions that are indispensable in the penetration process of spermatozoa through the molten zone. This is in accordance with the research of by Gyamera-Acheampong [5] and Gyamera-Acheampong and Mbikay [15], which states that the PCSK4 enzyme has an important role in reproductive physiology and that a disruption of expression or disturbance of proteolytic activity can cause fertility disorders.

In this test, spermatozoa given PCSK4 antibodies moved away from the oocyte extract compared with the controls whose spermatozoa were not treated. It is clear that the presence of PCSK4 antibodies can inhibit the binding of PCSK4 to its receptors in the initiation process of the acrosome reaction when the spermatozoa approach the oocyte by penetrating the pellucida zone of the ZP3 receptor. This agrees with other research stating that the PCSK4 enzyme plays a role in acrosome reactions through proteolytic processes and is supported also by the theory that the binding of the spermatozoa plasma membrane with the pellucida zones is the beginning of an acrosomal reaction that triggers intracellular signals to stimulate acrosomal exocytosis [24-26].

There were several limitations in this study. We do not yet know the antibody reversibility. We also cannot determine the antibody potency to penetrate the blood-testicular barrier, since in this study we only tested the antibody potency in vitro. Therefore, further research is needed to identify antibody reversibility and potency to penetrate the blood-testicular barrier in vivo.

\section{CONCLUSIONS}

The result of this study can be used as a basis to develop new immunocontraceptives targeted toward males. Several lines of evidence based on the study suggest that antibodies from induction of PCSK4 from spermatozoa may hinder fertilization: (1) PCSK4 enzymes on the plasma membrane of human spermatozoa are immunogenic and (2) PCSK4 antibodies created by induction of immunogenic PCSK4 from human spermatozoa may inhibit the binding of spermatozoa and oocytes through in vitro ligand binding receptor.

\section{CONFLICTS OF INTEREST}

The authors have nothing to disclose.

\section{REFERENCES}

1. Page ST, Amory JK, Bremner WJ. Advances in male contraception. Endocr Rev 2008;29:465-93. 
2. Gupta SK, Gupta N, Suman P, Choudhury S, Prakash K, Gupta $\mathrm{T}$, et al. Zona pellucida-based contraceptive vaccines for human and animal utility. J Reprod Immunol 2011;88:240-6.

3. Naz RK. Immunization with sperm antigens to induce contraception. In: Krause WKH, Naz RK. Immune infertility. Heidelberg, Berlin: Springer; 2009;197-207.

4. Naz RK. Human synthetic peptide vaccine for contraception targeting sperm. Arch Androl 2004;50:113-9.

5. Gyamera-Acheampong C. The physiology and biochemistry of the fertility enzyme Proprotein Convertase Subtilisin/Kexin type 4 [dissertation]. Ottawa: University of Ottawa; 2009.

6. Naz RK. Vaccine for contraception targeting sperm. Immunol Rev 1999;171:193-201.

7. Gyamera-Acheampong C, Sirois F, Denis NJ, Mishra P, Figeys D, Basak A, et al. The precursor to the germ cell-specific PCSK4 proteinase is inefficiently activated in transfected somatic cells: evidence of interaction with the BiP chaperone. Mol Cell Biochem 2011;348:43-52.

8. Kurniawan D. Characterization of antibodies resulting from protein induction of human spermatozoa membrane $20 \mathrm{kDa}$ (PM $20 \mathrm{kDa}$ ) [dissertation]. Malang: University Brawijaya; 2009.

9. Akmal M. Inhibin B potentially inhibits reversible spermatogenesis process through decreasing concentration and expression of follicle-stimulating hormone (FSH), CAMP responsive element modulator (CREM), protamine P2, and spermatozoa quality [dissertation]. Malang: University Brawijaya; 2011.

10. Aulani A. Analysis of glycosylated bovine zone pellucida-3 (bZP3dG) antibody induction for the development of contraceptive vaccines [dissertation]. Surabaya: University Airlangga; 2003.

11. Stanley J. Essentials of immunology and serology. New York: Thomson Learning; 2002.

12. Delves PJ, Seamus JM, Burton DR, Roitt IM. Roitt's essential immunology. 11 ed. Malden, Mass: Blackwell; 2006.

13. Goldberg AM. The principles of humane experimental technique: is it relevant today? ALTEX 2010;27:149-51.

14. Ferdowsian HR, Beck N. Ethical and scientific considerations regarding animal testing and research. PLoS One 2011;6: e24059.

15. Gyamera-Acheampong C, Mbikay M. Proprotein convertase subtilisin/kexin type 4 in mammalian fertility: a review. Hum Reprod Update 2009;15:237-47.

16. Bergeron F, Leduc R, Day R. Subtilase-like pro-protein convertases: from molecular specificity to therapeutic applications. J Mol Endocrinol 2000;24:1-22.

17. Torii S, Yamagishi T, Murakami K, Nakayama K. Localization of Kex2-like processing endoproteases, furin and PC4, within mouse testis by in situ hybridization. FEBS Lett 1993;316:12-6.

18. Seidah NG. The proprotein convertases, 20 years later. Methods Mol Biol 2011;768:23-57.

19. Chamley LW, Clarke GN. Antisperm antibodies and conception. Semin Immunopathol 2007;29:169-84.

20. Goldsby RA, Kindt TJ, Osborne BA. Immunology. California: W. H. Freeman and Company; 2000.

21. Parslow TG. Medical immunology. 10th ed. San Francisco: Mc Graw Hill; 2003;72-81.

22. Burkin HR, Miller DJ. Zona pellucida protein binding ability of porcine sperm during epididymal maturation and the acrosome reaction. Dev Biol 2000;222:99-109.

23. Esteves SC, Verza S Jr. Relationship of in vitro acrosome reaction to sperm function: an update. Open Reprod Sci J 2011;3: 72-84.

24. Gyamera-Acheampong C, Tantibhedhyangkul J, Weerachatyanukul W, Tadros H, Xu H, van de Loo JW, et al. Sperm from mice genetically deficient for the PCSK4 proteinase exhibit accelerated capacitation, precocious acrosome reaction, reduced binding to egg zona pellucida, and impaired fertilizing ability. Biol Reprod 2006;74:666-73.

25. Reid AT, Redgrove K, Aitken RJ, Nixon B. Cellular mechanisms regulating sperm-zona pellucida interaction. Asian J Androl 2011;13:88-96.

26. Nixon B, Aitken RJ, McLaughlin EA. New insights into the molecular mechanisms of sperm-egg interaction. Cell Mol Life Sci 2007;64:1805-23. 